\title{
Smart Grid Testbed using SCADA Software and Xbee Wireless Communication
}

\author{
Aryuanto Soetedjo \\ Dept. of Electrical Engineering \\ National Institute of Technology \\ Malang, Indonesia
}

\author{
Abraham Lomi \\ Dept. of Electrical Engineering \\ National Institute of Technology \\ Malang, Indonesia
}

\author{
Yusuf Ismail Nakhoda \\ Dept. of Electrical Engineering \\ National Institute of Technology \\ Malang, Indonesia
}

\begin{abstract}
This paper presents the development of Smart Grid testbed using SCADA software and Xbee wireless communication. The proposed testbed combines both the software simulation and the hardware simulation. The Winlog SCADA software is employed to implement the algorithm in the Smart Grid system. To communicate between nodes and the Smart Grid Center, the Xbee wireless communication is employed. The testbed is useful to test and verify the developed algorithms in the Smart Grid system. By using the hardware testbed, the more realistic simulation could be performed. While by using the software testbed, the complex model and algorithm could be implemented easily. The experimental results show that the proposed testbed works properly in simulating the continuous supply algorithm implemented in the Smart Grid system.
\end{abstract}

Keywords-SCADA; Smart Grid; wireless communication; Xbee DigiMesh; testbed

\section{INTRODUCTION}

The Smart Grid is a modern technology in the electrical power systems that delivering the electricity in a smart way, where the costumers are considered as the integral part of the system [1]. In the Smart Grid, the electricity and the information flow in two-way to achieve the efficient, clean, safe, reliable, and safe electrical power systems [2].

The Smart Grid integrates the sophisticated technology in electronics, communication, information, and electrical power systems. In general, the Smart Grid is divided into three major systems [2], i.e.: the smart infrastructure system, the smart management system, and the smart protection system. The smart infrastructure system consists of the smart energy subsystem, the smart information subsystem, and the smart communication subsystem. The smart management system deals with the energy efficiency, utility, cost, pricing, and optimization methods. The smart protection system provides the system reliability and failure protection, security and privacy.

The Smart Grid offers a wide research areas such as demand response, self healing, continuous supply, dynamic pricing, and power scheduling [3]. To develop and simulate those problems, the Smart Grid testbed is usually employed [3]-[14]. The Smart Grid testbeds based on the computer software were proposed in [4]-[7]. In [4], they compared several Smart Grid software simulators such as MatPower,

This work was supported by the Research Grant 2015 "Excellent Research on Higher Institution" from Directorate General of Higher Education, Ministry of Research and Technology and Higher Education, Republic of Indonesia (No. : SP DIPA-023.04.1.672453/2015).
PSAT, AMES, InterPSS, OpenPSS, GridLab-D. The simulators simulate the power system only. In [5], they proposed the SCORE (Smart Grid Common Open Research Emulator) that emulates both the power and communication networks. SCORE runs under Linux operating system. It consists of GUI (Graphical User Interface), Service Layer, Communication Module, and Power Module.

The real-time simulation of Smart Grid was proposed in [6]. They used the parallel computation combined by the advanced hardware that could handle hundreds of switches in real-time. The switching devices were modeled inside the FPGA (Field Programmable Gate Array) devices.

Our previous work [7] proposed the Smart Grid testbed using the SCADA (Supervisory Control and Data Acquisition) software configured on several computers as the client and the server. Each computer simulated the component of Smart Grid system such as the power resource and the demander. The TCP/IP protocol was adopted to communicate between the computers.

The benefits of software simulator are: a) No need high investment; b) It could simulate the complex algorithms easily; c) The simulation time is flexible. However the software simulator has the main drawback, i.e. it could not simulate the Smart Grid system in the realistic way. Some conditions in the software simulation may differ with the real implementation.

The Smart Grid testbeds using hardware simulator were developed in [3],[8]-[14]. In [3], they proposed the SmartGridLab that consits of the Intelligent Power Switch (IPS), the different types of power sources (photovoltaic, wind turbine, and utility grid), the energy demander, and the power meter. The testbed employed the 802.15.4 wireless mesh network for communicating between the nodes in the distributed mode, or between the nodes and the management center in the centralized mode. The IPS plays the important role for switching the power network, thus controlling the interconnection of power resources and demanders.

To reduce the costs of investment and installation of the renewable energy resources, the PV (photovoltaic) array and the wind turbine emulators were proposed in [8]. The PV emulator was simulated by regulating the DC output of power supply according to the I-V curve of the PV module. The wind turbine emulator consists of the wind speed emulator, the induction generator and the power inverter. 
The Smart Grid testbed using a wireless sensor network within a small building was developed in [9]. It consists of the application/dashboard, the server, the gateway, the electrical devices, and the information networks. The dashboard was designed to display electricity usages on a specific period. The server was used to collect and manage the electricity usages, and send the control messages for turning on/off the devices. The gateway provided the interface between the server and the wireless sensor networks. It consists of the PLC (Power Line Communication) gateway that uses the power line communication, and the Zigbee gateway that uses the Zigbee technology. The electrical devices consist of the smart meters, the wind generators, the PV generators, the electric vehicle chargers, the light controllers, and the smart outlets. The information networks consist of the PLC communication using the power line and the Zigbee technology developed based on Zigbee specification and IEEE 802.15.4.

The GSM/GPRS modems were used as the communication channel in the Smart Grid testbed developed by [10]. Data from the Smart Meter is transmitted to the Automatic Power Switches (APSs) as a SMS every three seconds. They proposed the distributed control scheme, where each APS decides its own control startegy. The centralized control scheme was proposed in [11]. In the centralized scheme, a control station was employed to collect data from the Smart Meters and to decide the control strategy for all APs.

The Smart Grid testbed was developed in [12] to simulate and monitor three-phase transmission line. The LabVIEW software was employed to display the measurement results such as the cable position, the inclination and vibration of the tower, and the frequency of the cable current. The testbed provided the unique tool for implementing the sensor networks on the transmission line.

The testbed for simulating the loads in the Smart Grid environments was proposed in [13]. They developed three types of loads, i.e. virtual, contextual and real loads. In the virtual load, the load is fully simulated by the computer. The contextual load is similar to the virtual load, but it is generated from the pre-defined value stored in the file. The real load consists of the real load connected to the Xbee module and the load connected to the PLC (Programmable Logic Controller). The testbed is focused for studying the demand side management system in the Smart Grid.

The power system components in the power system laboratories were integrated to establish the Smart Grid testbed [14]. The testbed consists of the main component usually available in the power system laboratories such as the generation station model, the transmission line model, the bus model, and the load model. The data acquisition devices were employed for monitoring and measurement. The network infrastructure was included to provide the communication network between devices.

Due to the complexity of the Smart Grid system, the testbeds are usually developed according to the particular function as described previously. In the hardware testbed, the application software is required for displaying the measurement results and for controlling the whole system. This application is usually developed using the programming language such as $\mathrm{C}++$, Java or using the popular software such as LabVIEW and Matlab. The programming language needs the high programming skill, while the LabVIEW and Matlab require the specific devices for interfacing with them. On the other hand, the SCADA software is very popular software for supervising and controlling the industrial processes. The SCADA software provides the tools for building the GUI and supports many communication protocols for interfacing with the field devices.

In this research, we exploit the capability of SCADA software for implementing the Smart Grid testbed. We extend our previous work [7] by developing the Smart Grid testbed that combining the software simulation and the real hardware. The wireless communication using Xbee protocol is employed to connect the nodes with the Smart Grid Control Center. The PV and wind turbine generators are simulated using the adjustable power supply. The DC-bus is adopted to connect the power resources and the demanders.

The rest of paper is organized as follows. Section 2 presents the architecture of the proposed Smart Grid testbed. Section 3 presents the implementation of SCADA software in the Smart Grid. The experimental results are described in Section 4. The conclusions are covered in Section 5.

\section{SMART GRID TeStBed ARchitecture}

The architecture of power network of the proposed Smart Grid testbed is illustrated in Fig. 1. It consists of three power resources (PV generator, Wind Turbine generator, and Utility Grid), two demanders (Home-1 and Home-2), and five Power Switches. They are connected by the 12 Volt DC-bus. The Power Switch plays an important role to manage the electricity flow. The Power Switch is controlled by the microcontroller that receives the command from the Smart Grid Center via the wireless communication.

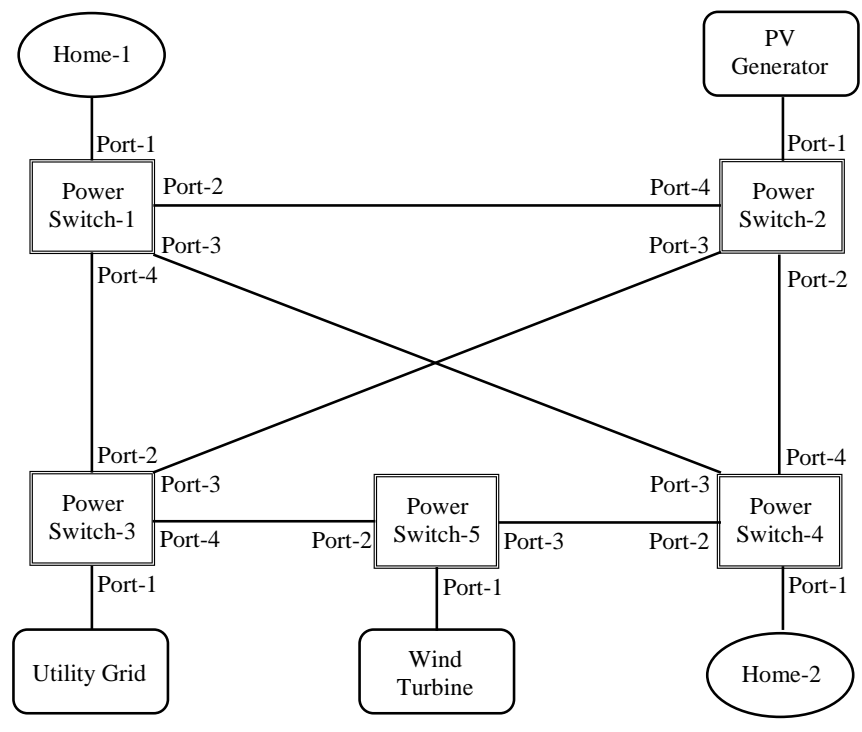

Fig. 1. Architecture of power network

Fig. 2 illustrates the architecture of information network in the Smart Grid testbed. As shown in the figure, there are five nodes and one center connected using Xbee communication. In 
each node, the Xbee module is attached on an embedded system using the Arduino microcontroller. The Smart Grid Center utilizes the SCADA software running on a personal computer. To connect with the nodes, the embedded system equipped with Xbee module is interfaced to the computer. The details explanation of the components are discussed in the following.

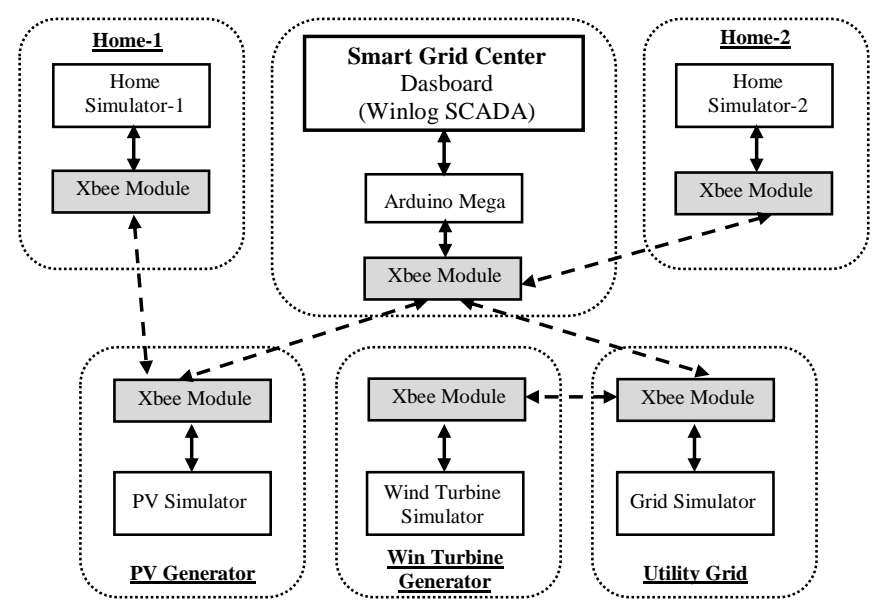

Fig. 2. Architecture of information network

\section{A. PV and Wind Turbine Simulators}

The PV simulator is designed by combining the software simulation and the adjustable power supply [15] as illustrated in Fig. 3. The simulator uses the Matlab Simulink to model the $\mathrm{I}-\mathrm{V}$ characteristic of the PV module. The output of the model is interfaced to the adjustable power supply controlled by the Arduino microcontroller. To provide the simple implementation, the microcontroller controls the position of servo motor which is attached to the potentiometer of the current controlled power supply. In addition to control the adjustable power supply, the Arduino microntroller reads the current and voltage of the system from the current and voltage sensors. It sends the electrical measurements to the Smart Grid Center via the Xbee module. At the same time, the Arduino controls the Power Switch according to the command sent by the Smart Grid Center.

The Wind Turbine simulator is designed in the similar way to the PV simulator. The difference lies on the software simulation, where the Simulink models the Wind Turbine generator instead of the PV generator. The hardware interfacing is similar to the PV simulator.

\section{B. Home Simulator}

The home simulator is used to simulate the demander or the load of Smart Grid system. The main component is the load unit consists of four lamps as illustrated in Fig. 4. The load is switched-on/off by the load relay controlled by the Arduino microcontroller. The status of load is defined by the user and simulated using SCADA software in the Smart Grid Center. Therefore, it is easy for the user to configure the load conditions. Further, this scheme could be used in the direct load control algorithm, which is one of the important features in the Smart Grid application.

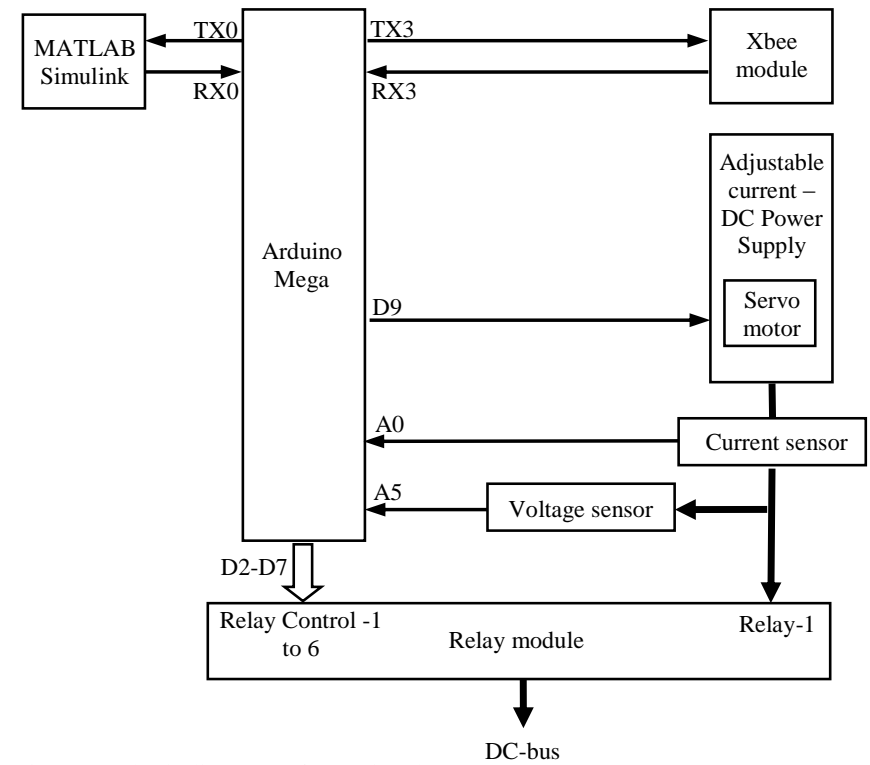

Fig. 3. Block diagram of PV Simulator

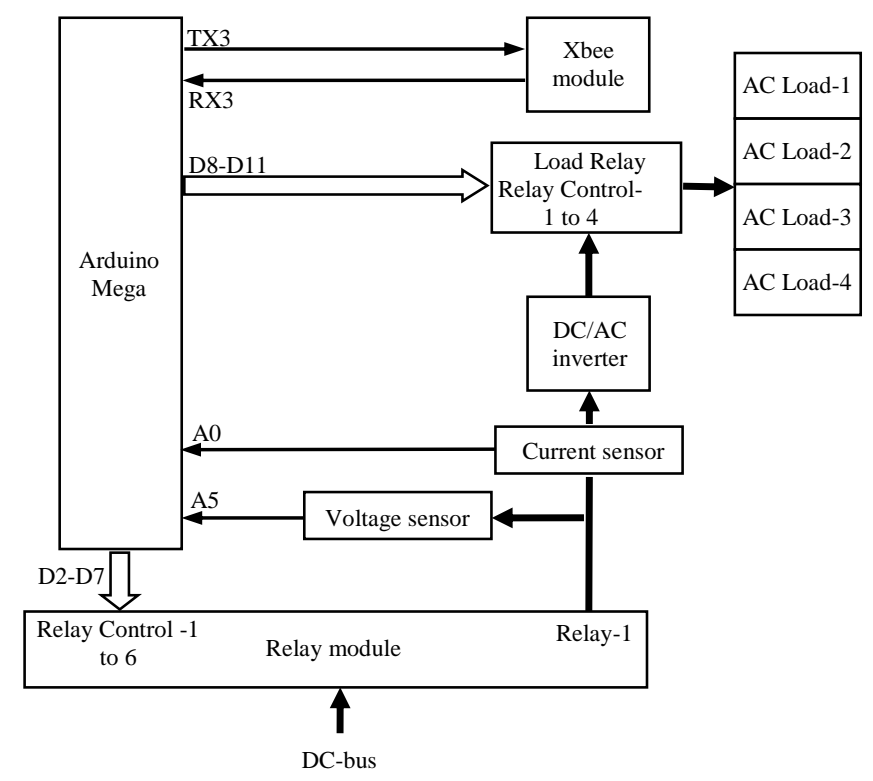

Fig. 4. Block diagram of home Simulator

\section{Smart Grid Center}

The proposed Smart Grid adopts the centralized scheme, thus the Smart Grid Center controls the whole procesess in the Smart Grid system. In the proposed testbed, the Winlog SCADA software ${ }^{1}$ is employed as the software tool for developing such system. The benefits of the SCADA software are; a) It is easy to develop the application based on GUI; b) It supports many communication protocols, thus it is easy to connect to the other devices.

The Smart Grid Center is implemented on a personal computer. To provide the flexibility and reduce the cost, the Arduino microcontroller is employed as the gateway between the computer and the nodes. The Arduino communicates to the

$\overline{{ }^{1} \text { http://www.sielcosistemi.com/en/products/winlog_scada_hmi/ }}$ 
computer using the serial communication, while it communicates to the other nodes using the Xbee wireless communication.

\section{Communication Protocols}

As discussed previously, there are two communication paths from the microcontroller to the computer, and the microcontroler to the nodes. Two different communication protocols are employed. The first protocol is the SCADA protocol, which is used to communicate between SCADA software in the Smart Grid Center and the Arduino microcontroller. In this research, the Modbus protocol is employed. Modbus protocol is an open protocol and widely used in the industrial applications.

The second protocol is the Xbee protocol used to communicate between Xbee modules. In the research, the Xbee DigiMesh $^{2}$ is employed, thus the mesh network as illustrated in Fig. 5 is adopted ${ }^{3}$. The DigiMesh provides the simple setup and the flexibility to expand the network. In our work, all Xbee modules are configured as the DigiMesh nodes (DN). It simplifies the implementation in the Smart Grid testbed system, such as the modules are interchangeable.

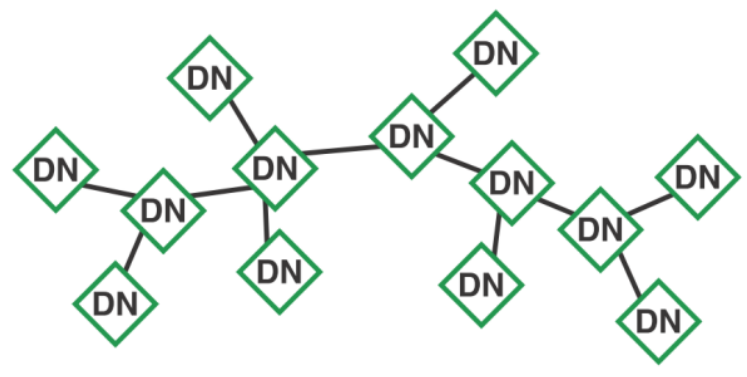

Fig. 5. DigiMesh network ${ }^{3}$

\section{IMPLEMENTATION OF SCADA SOFTWARE IN THE SMART GRID}

The major contribution of the paper is the implementation of SCADA software in the Smart Grid. The Winlog SCADA is the SCADA software usually used to develop the SCADA application. The objective of the research is to evaluate the capability of Winlog SCADA to be implemented in the Smart Grid application.

To implement the Winlog SCADA in the Smart Grid, four tools are utilized [7] : a) Configuration tool; b) Gate builder; c) Template builder; d) Code builder. The Configuration tool deals with the communication protocol to other devices, in this case the Modbus protocol. While the Gate builder is used to configure the variables of the process. In this system, the variables are used to define the current, voltage, power and relay status in the Smart Grid testbed.

The template builder is used to design the GUI of the Smart Grid system. Using this tool, the front panel/dashboard of the Smart Grid such as the visualization of the generators and the loads, and their corresponding electrical parameters, the lines representing the power network could be developed easily.

\footnotetext{
${ }^{2}$ http://www.digi.com/technology/digimesh/

${ }^{3}$ http://www.digi.com/pdf/wp_zigbeevsdigimesh.pdf
}

The Code builder is the tool for writing the scripts or program. In the research, we exploit the Code builder for implementing the Smart Grid algorithm. The simple and important algorithm is the one for solving the problem of continuous supply as desrcibed in the following.

Continuous supply is one of the simple Smart Grid feature that provides the continuous supply to the loads regardess of the power capacity of the generators or the generator's faulty. Fig. 6 illustrates the flowchart of this algorithm. The power network in Fig. 1 is used as the example.

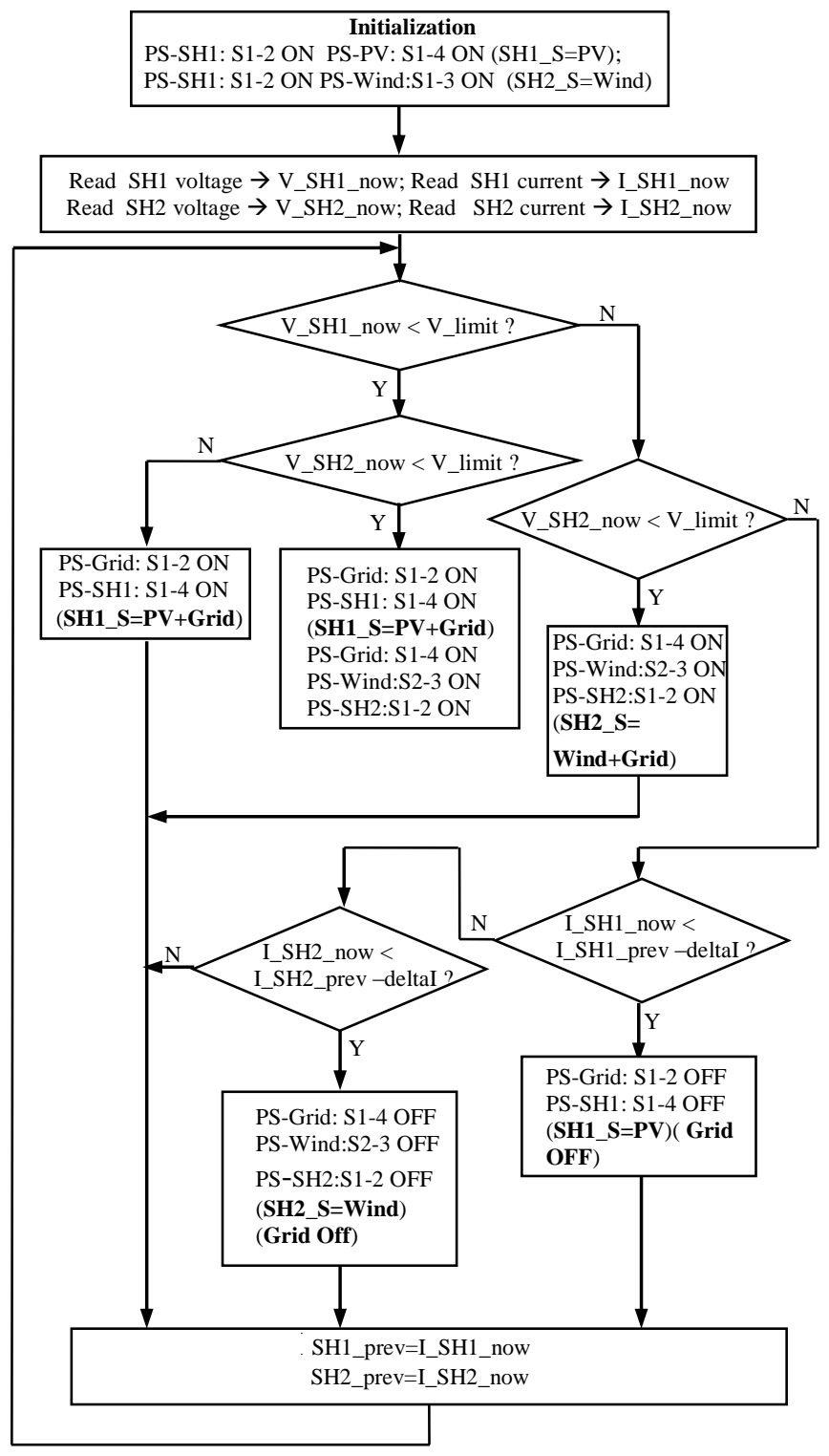

Fig. 6. Flowchart of the continuous supply algorithm

In the algorithm, it is assumed that the Home-1 gets the electricity from the PV as the primary source, and the Utility Grid as the secondary source. While the Home-2 gets the electricity from the Wind Turbine as the primary source, and the Utility Grid as the secondary source. When the power demanded by the Smarthome-1 (Smarthome-2) is lower than the power that could be delivered by the primary source, then 
only the primary source (PV or Wind Turbine) works, and called as the underload condition. Otherwise, in the overload condition, both the primary and secondary sources will work together.

The algorithm starts by intializing the relay status of the Power Switches. By default, the Port-1 and Port-2 of the PSSH1 (Power Switch of Smarthome-1) is connected by the relay S1-2 of PS-SH1; the Port-1 and Port-4 of the PS-PV (Power Switch of PV) is connected by the relay S1-4 of PS-PV; the Port-1 and Port-2 of the PS-SH2 (Power Switch of Smarthome2 ) is connected by the relay S1-2 of PS-SH2; the Port-1 and Port-3 of the PS-Wind (Power Switch of Wind Turbine) is connected by the relay S1-3 of PS-Wind. Thus the PV delivers the power to the Smarthome-1, while the Wind Turbine delivers the power to the Smarthome-2.

As shown in the flowchart, to detect the overload condition, the voltage of Smarthome-1 (Smarthome-2) is compared to a certain voltage limit. If the voltage is lower than this value, the overload condition occurs and the algorithm will switch-on the Power Switch appropriately to deliver the power from the secondary source. For example, when the Smarthome-1 suffers the overload condition, then the relay S1-2 of PS-Grid and the relay S1-4 of PS-SH1 will be switched-on. Thus the Smarthome-1 is supplied by both the PV and the Utility Grid.

The algorithm needs to check whether the overload condition is already completed and changes to the underload condition. This situation is determined by comparing the decreasing current on the Smarthome-1 (Smarthome-2) with a certain threshold. When the underload load condition occurs, the algorithm will switch-off the secondary source.

\section{EXPERIMENTAL RESULTS}

The proposed testbed was implemented in the software and hardware. The results of Smart Home simulator developed using Winlog SCADA is illustrated in Fig. 7. In the figure, the user could control the status of load by pushing the appropriate switch. Further, the operation could be performed automatically by writing the program in the Code builder, for example by time scheduling.

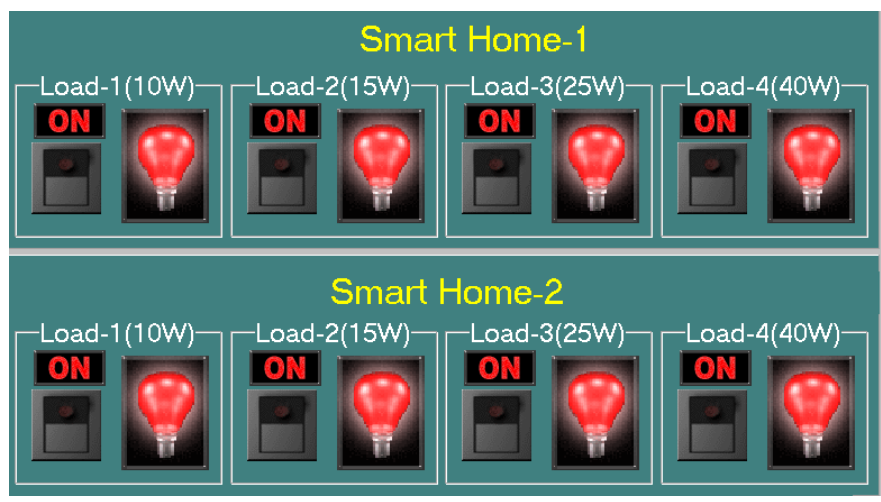

Fig. 7. Smarthome simulator developed using Winlog SCADA

To verify the proposed testbed, we conduct the experiments by varying the loads on the Smarthome-1 and the Smarthome-2 for simulating the overload and underload conditions as listed in Table 1. The power and voltage measurements on the Smart Grid testbed are illustrated in Fig. 8 and Fig. 9 respectively.

\section{TABLE I. LOAD PROFILE}

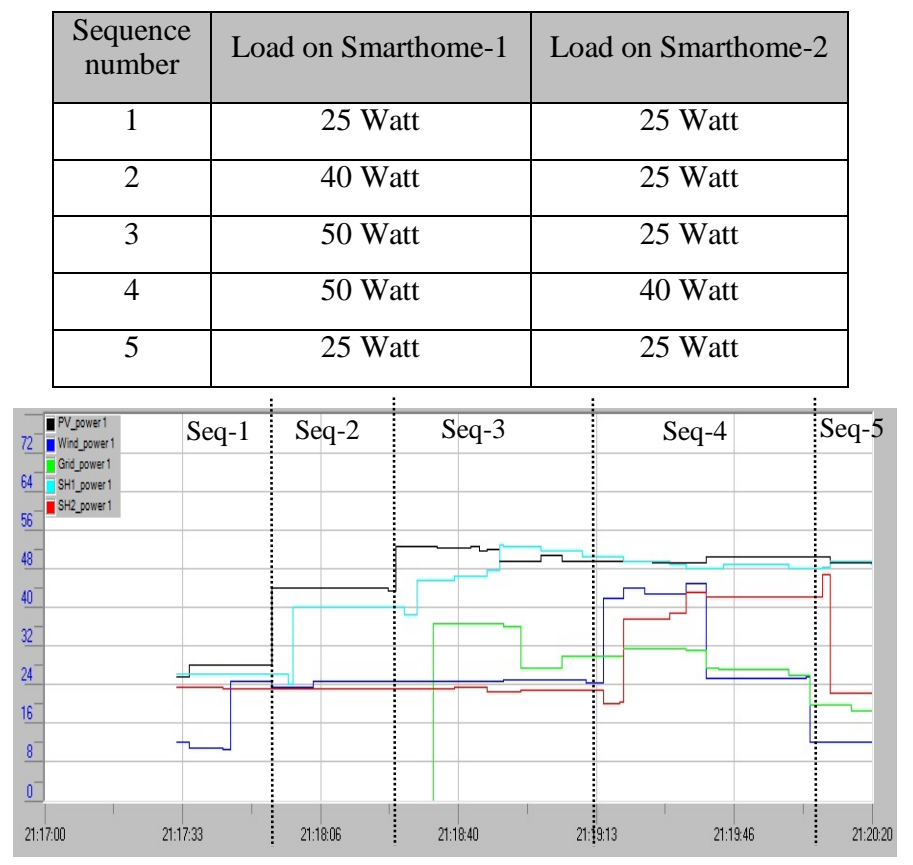

Fig. 8. Power measurement on the Smart Grid testbed

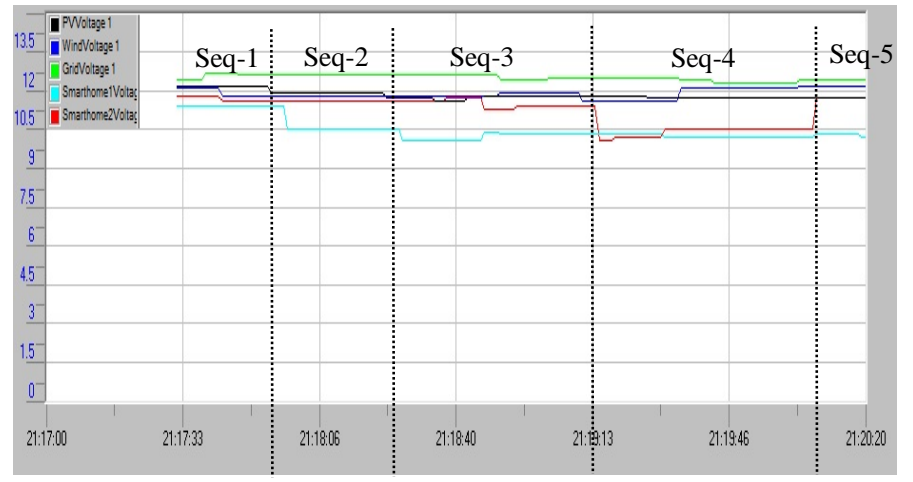

Fig. 9. Voltage measurement on the Smart Grid testbed

In the first sequence, the load of 25 Watt is applied to the Smarthome-1 and Smarthome-2. In this case, both the primary sources (PV and Wind Turbine) are sufficient to deliver the power, therefore the Utility Grid is switched-off from the grid as shown in Fig. 8. In the second sequence, the load on the Smarthome-1 is increased to 40 Watt, while the load on the Smarthome-2 remains the same. As shown in the figure, the PV is still sufficient to supply the power. The voltage on the Smarthome-1 as shown in Fig. 9 decreases, but it is still greater than the defined limit (i.e. 10.25 Volt). In the third sequence, the load on the Smarthome-1 is increased to 50 Watt. In this situation, the voltage drops below than 10.25 Volt, and the overload condition occurs. Therefore the system starts to switch-on the Utility Grid to deliver the power to the Smarthome-1. It needs the transition time about 30 second for recovery until the voltage increases above the voltage limit. 
In the fourth sequence, the load on the Smarthome-1 is not changed, while the load on the Smarthome- 2 is increased to 40 Watt. In this situation, the overload condition occurs and the system starts to switch-on the Utility Grid to deliver the power to the Smarthome-2. Therefore the Utility Grid delivers the power to both the Smarthome-1 and the Smarthome-2. In the fifth sequence, the load on the Smarthome-2 is decreased to 25 Watt, thus the condition changes to the underload. The system switches-off the Utility Grid for delivering the power to the Smarthome-2, but it still delivers the power to the Smarthome1. From the experiments, it is obtained that the power measurement on the Smarthome-1 (Smarthome-2) is not exactly same with the one on the PV (Wind Turbine) or the Utility Grid. This discrepancy is caused by the error in the sensor system for reading the electrical parameters.

The algorithm is also verified by observing the operation of relays on the Power Switches and the appearance of power lines on the SCADA front panel as illustrated in Fig. 10 and Fig. 11. Fig. 10 illustrates the appearance during the first sequence when the PV supplies to the Smarthome-1 and the Wind Turbine supplies to the Smarthome-2. As shown in the figure, the respective flow line changes to red color. The relay's status on the Power Switches are also indicated.

Fig. 11 illustrates the appearance during the third sequence when the Smarthome-1 is supplied by the PV and the Utility Grid, while the Smarthome-2 is supplied by the Wind Turbine only. From the figure, it is observed that two relays on the Power Switch of the Smarthome-1 are switched-on to perform this operation.

The above results show that the proposed testbed could be used to study and research the Smart Grid system, particulary the algorithm for manage the power network. Even thought the testbed is simple and small size, it could be expanded accordingly. It is worth to note that the Smart Grid algorithms could be implemented using the SCADA software.

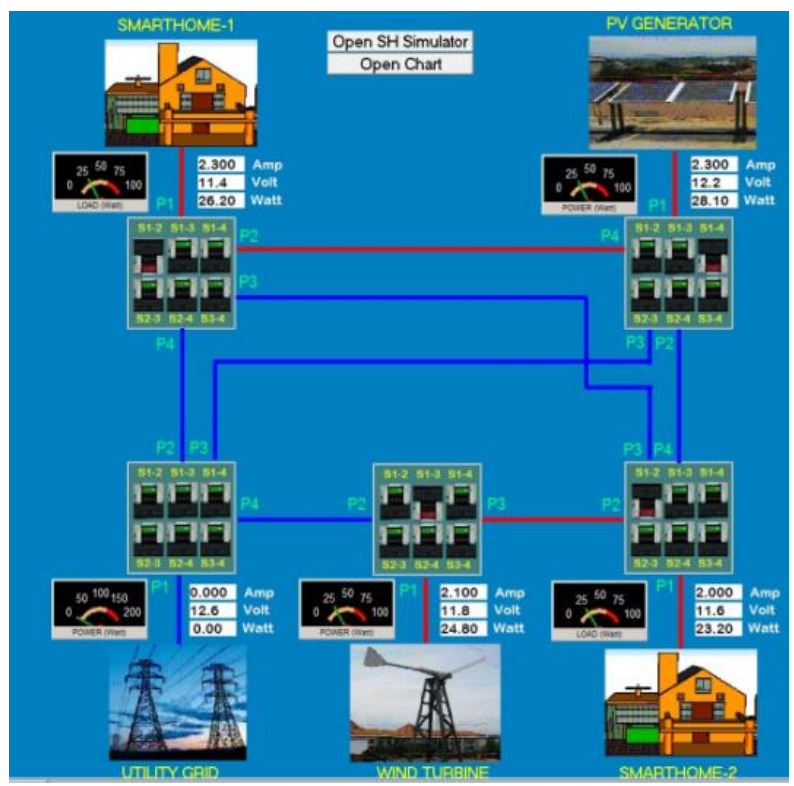

Fig. 10. Appearance of the Smart Grid front panel during the first sequence

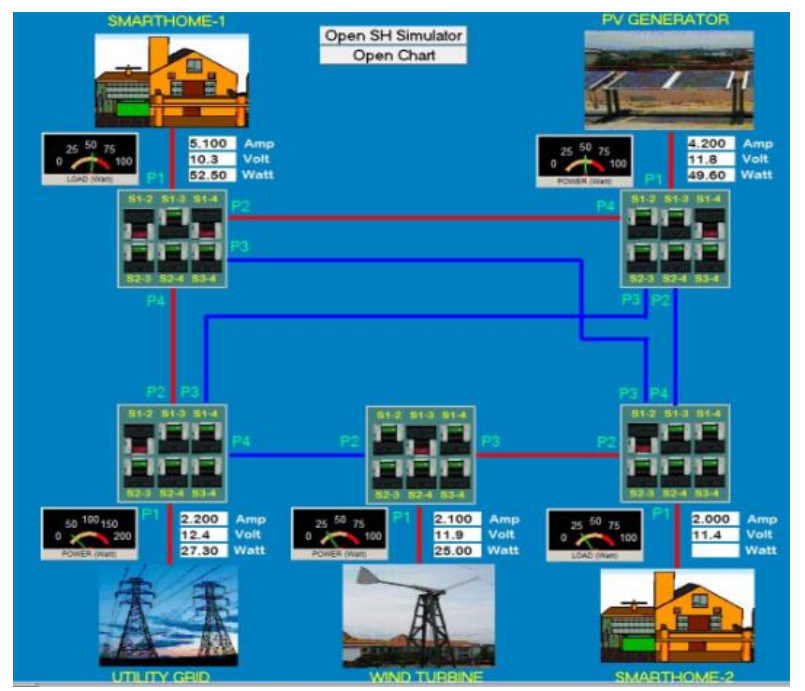

Fig. 11. Appearance of the Smart Grid front panel during the third sequence

Some of the hardware testbed simulator consists of the adjustable power supply with servo motor, the microcontroller, the Xbee module, and the Power Switch are illustrated in Fig. 12. The hardware module for each component/node is designed separately. The electrical power connections between them are conducted by the DC-bus cable. While the information connection is conducted wirelessly.

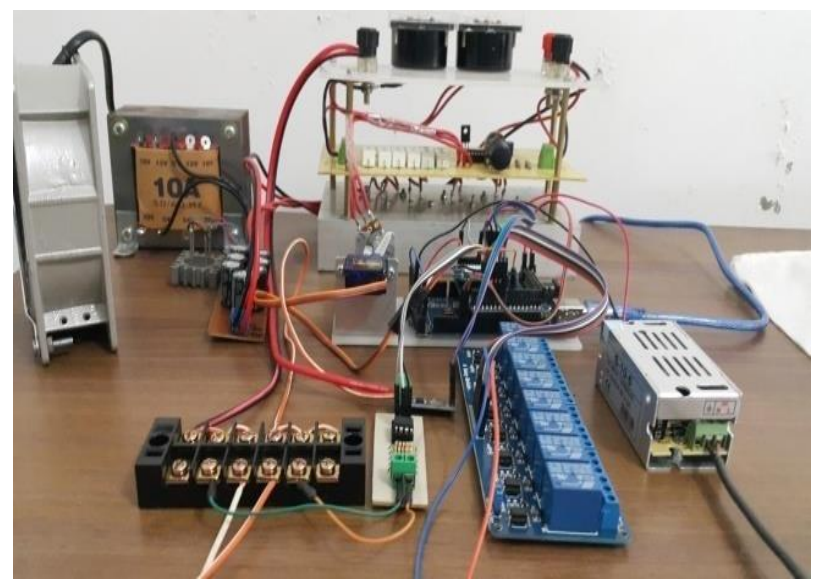

Fig. 12. PV simulator hardware.

\section{CONCLUSIONS}

The SCADA software is developed to implement the algorithm in the Smart Grid. The nodes on the Smart Grid testbed are communicated to the Smart Gird Center using the Xbee wireless communication. The power generators and the demanders are simulated using both the hardware and software. In the experiments, the system is tested to handle the problem of continuous supply when the power of the loads are varied. The developed Smart Grid testbed works appropriately in simulating the algorithm in the Smart Grid system. Further the front panel displayed on the Smart Grid Center could be used to help to operator/user to monitor and analyse the operation of the whole system. 
In future, the complex system will be developed. Further the sophisticated algorithms in the Smart Grid will be implemented.

\section{REFERENCES}

[1] P Siano, "Demand response and smart grid - a survey," Renewable and Sustainable Energy Reviews, Vol. 40, pp. 461-478, 2014.

[2] X. Fang, S. Misra, G. Xue, and D. Jang, "Smart Grid - The new and improved power grid: a survey," IEEE on Communication Surveys \& Tutorials, Vol. 14, No. 4, pp. 944-980, 2012.

[3] W.Z. Song, D. De, S. Tan, S.K. Das, and L. Tong, “ A wireless smart grid tesbed in lab," IEEE Wireless Communication, Vol. 19, No. 3, pp. 58-64, 2012.

[4] M. Pochacker, A. Sobe, and W. Elmenreich, "Simulating the smart grid," Proceedings of IEEE Grenoble PowerTech, Grenoble, France, June 16-20, 2013.

[5] S. Tan, W.Z. Song, Q. Dong, and L. Tong, "SCORE: Smart-Grid Common Open Reseacrh Emulator," Proceedings of IEEE Third International Conference on Smart Grid Communications, Tainan, Taiwan, November 5-8, 2012.

[6] F. Guo, L. Herrera, R. Murawski, E. Inoa, C.L. Wang, P. Beauchamp, E. Ekici, and. J. Wang, "Comprehensive real-time simulation of the smart grid," IEEE Transactions on Industry Applications, Vol. 49, No. 2, pp. 899-908, 2013.

[7] A. Soetedjo, A. Lomi, and Y.I. Nakhoda, "Simulation of smart grid using SCADA," Proceedings of International Conference on Quality in Research (QIR) 2015, Lombok, Indonesia, August 10-13, 2015.

[8] M. Shamshiri, C.K. Gan, and C.W. Tan, "A review of recent development in smart grid and micro-grid laboratories," Proceedings of
IEEE International Power Engineering and Optimization Conference, Melaka, Malaysia, June 6-7, 2012.

[9] K.S. Kim, H. Kim, T.W. Heo, Y. Doh, and J.A. Jun, "A smart grid tesbed using wireless sensor networks in a building," Proceedings of the Fifth International Conference on Sensor Technologies and Applications, French Riviera, France, August 21-27, 2011.

[10] S. Nithin, N. Radhika, and V. Vanitha, "Smart grid testbed based on GSM," Proceedings of International Conference on Communication Technology and System Design, Coimbatore, India, December 7-9, 2011.

[11] S. Nithin and N. Radhika, "Centralized control station for smart grid test bed based on Windows Embedded XP 2007 and Ebox 4861S," Proceedings of International Conference on Recent Trends in Information Processing and Computing, Kualu Lumpur, Malaysia, December 17-18, 2012.

[12] L.F. Cheung, K.S. Lui, K.K.Y. Wong, W.K. Lee, and P.W.T. Pong, "A laboratory-based three-phase smart grid sensor network testbed," Sensors and Materials, Vol. 26, No. 5, pp. 279-290, 2014.

[13] L. Gomes, et al., "Dynamic approach and tesbed for small and medium players simulation in smart grid environments," Proceedings of the International Federation of Automatic Control World Congress, Cape Town, South Africa, August 24-29, 2014.

[14] V. Salehi, A. Mazloomzadeh, J. Fernandez, J. Parra, and O. Mohammed, "Design and implementation of laboratory-based smart power system," Proceedings of the 2011 American Society for Engineering Education Annual Conference, Vancouver, Canada, June 26-29, 2011.

[15] A. Soetedjo, A. Lomi, Y.I. Nakhoda, and G.E. Hendroyono, "Simulating PV Generator using Simulink Interfaced to the Adjustable Power Supply for Smart Grid Testbed," unpublished. 